\title{
NONINVASIVE METHODS IN EVALUATION OF INFLAMMATORY BOWEL DISEASE: WHERE DO WE STAND NOW? AN UPDATE
}

\author{
Cansel Turkay, Benan Kasapoglu ${ }^{\mathrm{II}}$
}

doi: $10.1590 / \mathbf{S 1 8 0 7 - 5 9 3 2 2 0 1 0 0 0 0 2 0 0 0 1 5}$

\begin{abstract}
Turkay C, Kasapoglu B. Noninvasive methods in evaluation of inflammatory bowel disease: where do we stand now? An updat. Clinics. 2010;65(2):221-31.

The inflammatory bowel diseases, consisting of Crohn's disease, ulcerative colitis and indeterminate colitis, are distinguished by idiopathic and chronic inflammation of the digestive tract. The distinction between inflammatory bowel diseases and functional bowel disorders, such as irritable bowel syndrome, can be complex because they often present with similar symptoms. Rapid and inexpensive noninvasive tests that are sensitive, specific and simple are needed to prevent patient discomfort, delay in diagnosis, and unnecessary costs. None of the current commercially available serological biomarker tests can be used as a stand-alone diagnostic in clinics. Instead, these are used as an adjunct to endoscopy in diagnosis and prognosis of the disease.Along these lines,, fecal lactoferrin and calprotectin tests seem to be one step further from other tests with larger number of studies, higher sensitivity and specificity and wider availability.
\end{abstract}

KEYWORDS: Inflammatory bowel disease; Diagnosis; Serology; Fecal markers.

\section{INTRODUCTION}

The inflammatory bowel diseases (IBD), consistsCrohn's disease (CD), ulcerative colitis (UC) and indeterminate colitis (IC) which are distinguished by idiopathic and chronic inflammation of the digestive tract. These diseases have been shown to result from an aberrant innate and acquired immune response to commensal microorganisms in genetically susceptible individuals. ${ }^{1}$ Currently, the incidence of IBD is increasing worldwide, especially in Northern Europe and North America. Ethnic origin, lifestyle, presence of susceptibility regions on at least 12 chromosomes and geographical factors play a central role in the epidemiology of these diseases. ${ }^{2,3}$

\footnotetext{
${ }^{\text {I }}$ Department of Gastroenterology, Fatih University Medical School - Ankara, Turkey

II Department of Internal Medicine, Fatih University Medical School - Ankara, Turkey

Email: benankasapoglu@ hotmail.com

Tel.: 90-0312-4829166 or 2126262

Received for publication on August 28, 2009

Accepted for publication on November 05, 2009
}

The distinction between IBD and functional bowel disorders, such as irritable bowel syndrome (IBS), can be complex since they often present with similar symptoms, including abdominal distention, pain and diarrhea, and therefore, invasive and expensive tests may be necessary. The diagnoses of IBDs depend on the clinical findings after radiological, endoscopic and histological examinations. Although the division between $\mathrm{UC}$ and $\mathrm{CD}$ is generally clear, indeterminate colitis is present in 10-20\% of patients with isolated colitis. ${ }^{4}$ Noninvasive tests for both the diagnosis and follow-up of IBD have gained increasing attention. Rapid and inexpensive noninvasive tests that are sensitive, specific and simple are necessary to prevent patient discomfort, delay in diagnosis and unnecessary costs. The biomarkers of IBD, including serological tests, fecal markers and genetically predisposed gene polymorphisms, are tools for disease diagnosis, estimation of activity, follow-up and disease prognosis. ${ }^{5-7}$ Moreover, in conjunction with the development of imaging techniques, such techniques as imaging biomarkers with ultrasound, magnetic resonance imaging (MRI), X-ray, computer tomograghy (CT), position emission tomography (PET) 
and single photon emission computed tomography (SPECT) are also defined. ${ }^{8}$

Therefore, the purpose of the present study was to critically review the current literature on the diagnosis and follow-up of inflammatory bowel diseases. We systematically searched Medline and the Cochrane Database, with no language restrictions, for studies of humans on the topic of IBD diagnosis that were published between January 1960 and August 2009. The key words inflammatory bowel diseases, Ulcerative colitis, Crohn's disease, fecal calprotectin, lactoferrin, serology and their equivalent Medical Subject Heading terms were used.

\section{SEROLOGICAL MARKERS}

Serological testing has been used for many years in the diagnosis of IBDs. Serological biomarkers are primarily produced upon intestinal exposure to normal commensal bacteria ${ }^{9,10}$ and might reflect a disregulated immune inflammatory response. ${ }^{11,12}$ Most of the major serological biomarkers utilized in IBD clinics are antibodies to microbial antigens, including yeast oligomanna (antiSaccharomyces cerevisiae, ASCA), bacterial outer membrane porin $\mathrm{C}(\mathrm{OmpC})$, Pseudomonas fluorescens bacterial sequence I2 (anti-I2) and, most recently, bacterial flagellin (CBir 1). ${ }^{13}$

All of these antibodies are predominantly found in CD but are not found in UC, except ASCA, which is identified in $5 \%$ of UC patients. On the other hand, the human antibody, perinuclear antineutrophil cytoplasm antibody (pANCA) is considered to be an autoantibody, although the specific antigenic stimulation for its production remains imprecise. PANCA has currently been found in up to $70 \%$ of patients with UC and in up to $20 \%$ of patients with $\mathrm{CD} .{ }^{14}$

Five new anti-glycan antibodies anti-chitobioside IgA (ACCA), anti-laminaribioside IgG (ALCA), antimanobioside $\operatorname{IgG}$ (AMCA) and antibodies against chemically synthesized $(\Sigma)$ two major oligomannose epitopes, Man $\alpha-1,3$ Man $\alpha-1,2$ Man ( $\Sigma$ Man3) and Man $\alpha-1,3$ Man $\alpha-1,2$ Man $\alpha-1,2$ Man ( $\Sigma$ Man4) are recognized recently. ${ }^{13,15}$ Since these new biomarkers have been shown to be present only in IBD, they might signify an intestinal inflammation that is specific to UC or CD. Moreover, these antibodies have been primarily studied in $\mathrm{CD}$ and have a high specificity but poor sensitivity.

Joossens et al. investigated 86 families from Belgium and Northern France to test whether a combination of CDassociated genes and/or antibody responses to microbial antigens might be valuable in identifying healthy relatives at risk. Genetic (NOD2, NOD1, TLR4, CARD8) and new serologic markers (ASCA, ACMA, ALCA, ACCA,
ASigmaMA, OmpC, CBir1, I2) were analyzed in all of the subjects. After a follow-up of 54 months, the authors found that there was an additive risk for CD in subjects from multi-case families per additional affected relative and per additional positive antibody, and this was independent of NOD2 genetic marker. ${ }^{16}$ These new antibodies might be important in complicated disease phenotype and might predict the need for surgery.

Recently, Mokrowiecka studied 125 IBD patients (71 UC, 31CD and 23 IC) and 45 patients with functional intestinal disorders to determine the accuracy of pANCA and ASCA in patients with IBD subgroups. In UC patients, the prevalence of pANCA was $68 \%$, which was significantly higher than in CD (29\%). ASCA were found significantly more often in CD (80.6\%) than in UC patients (26.8\%). The sensitivity, specificity, positive predictive value (PPV) and negative predictive value (NPV) of pANCA for UC diagnosis were $68 \%, 84 \%, 75 \%$ and $78 \%$, respectively, and of ASCA for CD diagnosis were $81 \%, 78 \%, 45.5 \%$ and $95 \%$, respectively. Moreover, the combined use of these two markers provided changes in diagnostic accuracy, such that for pANCA+/ASCA- in UC the sensitivity, specificity, PPV and NPV of results were $42 \%, 100 \%, 100 \%$ and $43 \%$, respectively, and for pANCA-/ASCA+ in CD the results were $52 \%, 98.6 \% 94 \%$ and $82 \%$, respectively. The authors concluded that the specificity of these combined serological markers tended to be higher than their sensitivity, and thus, these markers are more useful in the differentiation of IBD subtypes than in screening the population. ${ }^{17}$

Anand et al. evaluated 98 adults with IBD and found that ASCA and pANCA had a $32 \%$ sensitivity and $100 \%$ specificity for Crohn's disease, while there was a $50 \%$ sensitivity and $90 \%$ specificity for UC. ${ }^{18}$

Interestingly, in another study, the presence of ASCA was found to be associated not only with the existence of Crohn's disease but also with markers of disease severity and oral involvement. ${ }^{19}$

Two novel immunoglobulin A (IgA) cell wall polysaccharide antibodies, anti-laminarin (anti-L) and anti-chitin (anti-C), were analyzed during the diagnosis and phenotype differentiation of Crohn's disease and UC. A cohort of 818 individuals with IBD (517 CD and 301 UC) were analyzed for seven anti-glycan antibodies (gASCA (anti-Saccharomyces cerevisiae) IgG, gASCA IgA, anti-chitobioside (GlcNAc(beta1,4)GlcNAc(beta)), antilaminaribioside (Glc(beta1,3)Glb(beta)), anti-mannobioside (Man(alpha1,3)Man(alpha)), anti-L and anti-C) and for pANCA. ${ }^{20}$ The authors found that all of the glycan markers were specific for and more prevalent in CD than in UC and, additionally, that gASCA IgG and IgA best differentiated CD from UC, followed by anti-L. The authors concluded that 
anti- $\mathrm{L}$ and anti-C improved the ability to differentiate between $\mathrm{CD}$ and UC and that these antibodies were independently associated with a more aggressive $\mathrm{CD}$ phenotype. Chen et al. described the use of a whole $E$. coli proteome microarray as a novel high-throughput approach to screen and identify new serological biomarkers for IBD. With the use of protein arrays containing 4,256 E. coli K12 proteins, Chen et al. have identified novel sets of serological biomarkers for the diagnosis of IBD that have a $>80 \%$ overall accuracy and sensitivity in differentiating $\mathrm{CD}$ from UC. ${ }^{21}$

It is important to keep in mind that the diagnostic value of serological biomarkers can show a discrepancy among different ethnic or geographic groups. For instance, both ASCA and pANCA were found to be less sensitive in Chinese and Japanese patients, while the positivity of pANCA was shown to be higher in Mexican-American UC patients. 22,23

It is also essential to emphasize that none of the current commercially available serological biomarker tests can be used alone as a diagnostic in clinics. Instead, they are used in addition to endoscopy in diagnosis and prognosis of the disease. Whether or not serologic markers have a role in screening for IBD remains controversial. However, due to the generally low sensitivity and specificity of these markers for distinguishing IBD from non-IBD, they are generally not recommended for use as a screening test. As a consequence, specific and sensitive IBD serologic biomarkers are desired, as well as future studies to evaluate the efficacy of current and newly identified biomarkers.

\section{BLOOD INFLAMMATORY MARKERS}

Erythrocyte sedimentation rate (ESR), white blood cell count (WBC) and C-reactive protein (CRP) are known to be good predictors of disease activity in IBD. CRP, with its short half-life, becomes rapidly elevated soon after the onset of the inflammatory process and decreases after its resolution. Moreover evaluating CRP is simple, easily available and inexpensive. ESR is also inexpensive and easily available, but since it has a longer half-life it differs from CRP and causes a prolonged latency period after changes in IBD activity. In clinical practice, because ESR, WBC and CRP are non-specific, they sometimes are not helpful for the differential diagnosis and follow-up of IBD. ${ }^{24,25}$

In addition, ESR has been found to be more reliable to be correlated with the disease activity. ${ }^{26}$ The pro-inflammatory cytokines (TNF-alpha, IL-1beta IL-6, and IL-8) are also found to be elevated in IBD patients. ${ }^{27}$ However, these are not widely available and are not specific for intestinal inflammation.

\section{FECAL MARKERS}

Fecal markers comprise a heterogeneous group of substances that either pour out from, or are generated by, the inflamed intestinal mucosa. ${ }^{28}$ The fecal excretion of Indium 111-labeled leukocytes is considered to be the gold standard fecal marker of inflammation, with a sensitivity of $97 \%$ for the diagnosis of IBD. ${ }^{29}$ Even though the use of radiolabeling techniques remains very important for research studies, they are not recommended for routine use due to high cost, exposure to radiation and the need for 4 days of fecal collection.

Fecal levels of Alpha1 1-antitrypsin, which is a protease inhibitor produced by the liver, macrophages and intestinal epithelium, are a useful indicator of IBD. Random levels of fecal Alpha1-antitrypsin levels are revealed to be useful in measuring $C D$ activity, while testing a 72-h fecal clearance of Alpha1-antitrypsin is a useful method for quantification of intestinal protein loss. ${ }^{30,31}$ Although fecal $\alpha 1$-antitrypsin has been generally accepted as a useful marker of IBD, it is not routinely available and costeffective.

Fecal excretion of another serum anti-proteinase, alpha2macroglobulin, is also increased in IBD patients. The levels of alpha2-macroglobulin in the feces have a positive relationship with the activity index in $\mathrm{CD}$ but not in subjects with UC. ${ }^{32}$

The neutrophil-derived proteins, lysozyme, myeloperoxidase, calprotectin, lactoferrin, and PMNelastase, are generally elevated in the feces of IBD patients. ${ }^{33-39}$ However, fecal lactoferrin and calprotectin are more appropriate for the differentiation of chronic IBD from IBS, and their increased levels show a positive relationship with the severity of inflammation. Some recent studies that deal with the relationship of fecal markers in IBD are summarized in Table 1. ${ }^{48-52,59,66,68-74}$

\section{Fecal Lactoferrin}

Lactoferrin is an $80-\mathrm{kDa}$ iron-binding glycoprotein and a major component of the secondary granules of polymorphonuclear neutrophils. In intestinal inflammation, leukocyte infiltration of the mucosa causes a rise in lactoferrin concentration in the feces. Lactoferrin has antibacterial activity and is resistant to proteolysis in the feces. Lactoferrin can be detected using simple and inexpensive techniques since it has an excellent stability in the feces for 4 days since a commercial ELISA has been developed and is now widely available. A negative fecal lactoferrin test simply means that there is an absence of significant neutrophilic intestinal inflammation..$^{40,41}$ 
Table 1 - Some recent studies about the fecal markers in the evaluation of IBD

\begin{tabular}{|c|c|c|c|}
\hline Study & Aim & Patient & Result \\
\hline Erbayrak et al ${ }^{48}$ & $\begin{array}{l}\text { To investigate the role of FC } \\
\text { in evaluating IBD activity }\end{array}$ & $\begin{array}{l}65 \mathrm{IBD} \text { and } 20 \mathrm{IBS} \\
\text { patients }\end{array}$ & $\begin{array}{l}\text { ESR, CRP, and FCP values were higher } \\
\text { in the IBD patients than in the control } \\
\text { group, while the hgb level was lower in } \\
\text { the IBD group. No statistically significant } \\
\text { differences in FCP levels were detected } \\
\text { between UC and CD patients. }\end{array}$ \\
\hline
\end{tabular}

\begin{tabular}{ll}
\hline Schoepfer et $^{4 \mathbf{l}^{49}}$ & $\begin{array}{l}\text { To evaluate the correlation } \\
\text { between endoscopic disease } \\
\text { activity and FC, CAI, CRP, }\end{array}$
\end{tabular}
and blood leukocytes in UC

$134 \mathrm{UC}$ patients and 48
controls

FC levels were significantly lower in UC patients with inactive disease. The overall accuracy for the detection of endoscopically active disease was $89 \%$ for FC, $73 \%$ for CAI, $62 \%$ for elevated CRP, and $60 \%$ for leukocytosis.

\begin{tabular}{|c|c|}
\hline Eder et $\mathbf{a l}^{50}$ & $\begin{array}{l}\text { To evaluate the diagnostic } \\
\text { utility of the assessment of } \\
\text { FC concentration in patients } \\
\text { with CD. }\end{array}$ \\
\hline
\end{tabular}

$31 \mathrm{CD}$ and 12 IBS patients

Mean FC concentration in CD group was statistically higher than among IBS patients. There was a positive correlation between FC concentration and CRP, and negative--with hemoglobin concentration.

\begin{tabular}{ll}
\hline Gisbert et al $^{\mathbf{5 1}}$ & $\begin{array}{l}\text { To determine the role of FC } \\
\text { and FL in the prediction of } \\
\text { IBD relapses }\end{array}$
\end{tabular}

D'Inca et al $^{\mathbf{5 2}} \quad$ To assess the role of $\quad 97 \mathrm{UC}$ and $65 \mathrm{CD}$
calprotectin tests in predicting clinical relapse in IBD patients.

$97 \mathrm{UC}$ and $65 \mathrm{CD}$

Sensitivity and specificity to predict relapse of IBD for FC ( $>150$ microg/g) and FL were $69 \%$ and $69 \%$, and $62 \%$ and $65 \%$, respectively.

Conclusion

FC was found to be strongly associated with colorectal inflammation indicating organic disease.

FC was the only marker that reliably discriminated inactive from active disease, emphasizing its usefulness for activity monitoring. The assessment of FC concentration may be useful in differential diagnoses of $\mathrm{CD}$ and monitoring patients with $\mathrm{CD}$.

FC and FL determination may be useful in predicting impending clinical relapse-in both CD and UC patients.

A significant correlation emerged between Measuring calprotectin may a positive $\mathrm{FC}$ test and the probability of relapse in UC patients. In CD patients, only cases of colonic $\mathrm{CD}$ showed a significant correlation between a positive FC test and the probability of relapse.

\begin{tabular}{lll}
\hline Jeffrey et al $^{\mathbf{5 9}}$ & To investigate fecal tumor & 94 controls and 105 \\
M2-PK in the differentiation & outpatients of whom 14 \\
of functional from organic & were diagnosed with \\
bowel disease. & $\begin{array}{l}\text { organic bowel disease } \\
\text { later }\end{array}$
\end{tabular}

Sensitivity and specificity, for diagnosis of organic bowel disease were $93 \%, 92 \%$ for FC and 67\%, 88\% for tumor M2-PK, respectively.

help to identify UC and colonic CD patients at higher risk of clinical relapse.

Tumor M2-PK is able to differentiate organic from functional bowel disease but has a lower sensitivity, specificity, and predictive value than FC.

\begin{tabular}{|c|c|}
\hline Wagner et al ${ }^{66}$ & $\begin{array}{l}\text { To evaluate FC as a marker of } 27 \mathrm{UC} \text { and } 11 \mathrm{CD} \\
\text { treatment outcome of relapse patients } \\
\text { of IBD and, to compare FC } \\
\text { with fecal myeloperoxidase } \\
\text { (MPO) and fecal eosinophil } \\
\text { protein X (EPX) }\end{array}$ \\
\hline
\end{tabular}

\begin{tabular}{ll}
\hline Lamb et $\mathbf{a l}^{68}$ & $\begin{array}{l}\text { To evaluate FC and FL in } \\
\text { identifying CD recurrence in } \\
\text { symptomatic patients after }\end{array}$
\end{tabular}
ileocaecal resection

117 CD patients

(1)

$\begin{array}{ll}\text { 11 CD } & \text { A normalised FC level at the end of the } \\ & \text { study predicted a complete response in } \\ & \text { 100\% patients, whereas elevated FC level } \\ & \text { predicted incomplete response in 30\%. } \\ & \text { Normalised MPO or EPX levels predicted } \\ & \text { a complete response in 100\% and 90\% } \\ & \text { of the patients, respectively. However, } \\ \text { elevated MPO or EPX levels predicted } \\ \text { incomplete response in 23\% and 22\%, } \\ \text { respectively. }\end{array}$

\begin{tabular}{|c|c|c|}
\hline Ashorn et al ${ }^{69}$ & $\begin{array}{l}\text { To identify new noninvasive } \\
\text { test combinations for } \\
\text { characterization of IBD in } \\
\text { children and adolescents } \\
\text { by comparing serological } \\
\text { responses to microbial } \\
\text { antigens. }\end{array}$ & $\begin{array}{l}73 \text { children who } \\
\text { underwent endoscopies } \\
\text { because of suspicion } \\
\text { of IBD and IBD was } \\
\text { diagnosed in } 60 \text { patients } \\
\text { (18 CD, } 36 \text { UC, 6 IC). }\end{array}$ \\
\hline
\end{tabular}
antigens.
A normalised FC level has the potential to be used as a surrogate marker for successful treatment outcome in IBD patients. FC and MPO provide superior discrimination than EPX in IBD treatment outcome.

FC and FL are non-invasive tests that can help to identify disease recurrence in symptomatic postoperative patients.
A normalised FC level at the end of the
study predicted a complete response in
$100 \%$ patients, whereas elevated FC level
predicted incomplete response in $30 \%$.
Normalised MPO or EPX levels predicted
a complete response in $100 \%$ and $90 \%$
of the patients, respectively. However,
elevated MPO or EPX levels predicted
incomplete response in $23 \%$ and $22 \%$,
respectively.
In patients with mild to moderately
clinically active disease, FC and FL
identified individuals with and without
recurrent IBD. Faecal markers were more
accurate at predicting clinical disease
activity than CRP, platelet count or
endoscopic appearance.
FC levels were elevated more frequently
in IBD patients ( $89 \%$ vs $9 \%$ ). ASCA
antibodies were detected in $67 \%$ of
patients with CD, The combination of
the measurements of FC and serological
responses to microbial antigens (ASCA,
I2, and OmpW) identified 100\% of CD
patients (specificity $36 \%$ ) and $89 \%$ of UC
patients (specificity $36 \%$ ).

A normalised FC level at the end of the
study predicted a complete response in
$100 \%$ patients, whereas elevated FC level
predicted incomplete response in $30 \%$.
Normalised MPO or EPX levels predicted
a complete response in $100 \%$ and $90 \%$
of the patients, respectively. However,
elevated MPO or EPX levels predicted
incomplete response in $23 \%$ and $22 \%$,
respectively.
In patients with mild to moderately
clinically active disease, FC and FL
identified individuals with and without
recurrent IBD. Faecal markers were more
accurate at predicting clinical disease
activity than CRP, platelet count or
endoscopic appearance.
FC levels were elevated more frequently
in IBD patients (89\% vs $9 \%$ ). ASCA
antibodies were detected in $67 \%$ of
patients with CD, The combination of
the measurements of FC and serological
responses to microbial antigens (ASCA,
I2, and OmpW) identified 100\% of CD
patients (specificity $36 \%$ ) and $89 \%$ of UC
patients (specificity $36 \%$ ).

A normalised FC level at the end of the
study predicted a complete response in
$100 \%$ patients, whereas elevated FC level
predicted incomplete response in $30 \%$.
Normalised MPO or EPX levels predicted
a complete response in $100 \%$ and $90 \%$
of the patients, respectively. However,
elevated MPO or EPX levels predicted
incomplete response in $23 \%$ and $22 \%$,
respectively.
In patients with mild to moderately
clinically active disease, FC and FL
identified individuals with and without
recurrent IBD. Faecal markers were more
accurate at predicting clinical disease
activity than CRP, platelet count or
endoscopic appearance.
FC levels were elevated more frequently
in IBD patients (89\% vs $9 \%$ ). ASCA
antibodies were detected in $67 \%$ of
patients with CD, The combination of
the measurements of FC and serological
responses to microbial antigens (ASCA,
I2, and OmpW) identified 100\% of CD
patients (specificity $36 \%$ ) and $89 \%$ of UC
patients (specificity $36 \%$ ).

A normalised FC level at the end of the
study predicted a complete response in
$100 \%$ patients, whereas elevated FC level
predicted incomplete response in $30 \%$.
Normalised MPO or EPX levels predicted
a complete response in $100 \%$ and $90 \%$
of the patients, respectively. However,
elevated MPO or EPX levels predicted
incomplete response in $23 \%$ and $22 \%$,
respectively.
In patients with mild to moderately
clinically active disease, FC and FL
identified individuals with and without
recurrent IBD. Faecal markers were more
accurate at predicting clinical disease
activity than CRP, platelet count or
endoscopic appearance.
FC levels were elevated more frequently
in IBD patients (89\% vs $9 \%$ ). ASCA
antibodies were detected in $67 \%$ of
patients with CD, The combination of
the measurements of FC and serological
responses to microbial antigens (ASCA,
I2, and OmpW) identified 100\% of CD
patients (specificity $36 \%$ ) and $89 \%$ of UC
patients (specificity $36 \%$ ).

A normalised FC level at the end of the
study predicted a complete response in
$100 \%$ patients, whereas elevated FC level
predicted incomplete response in $30 \%$.
Normalised MPO or EPX levels predicted
a complete response in $100 \%$ and $90 \%$
of the patients, respectively. However,
elevated MPO or EPX levels predicted
incomplete response in $23 \%$ and $22 \%$,
respectively.
In patients with mild to moderately
clinically active disease, FC and FL
identified individuals with and without
recurrent IBD. Faecal markers were more
accurate at predicting clinical disease
activity than CRP, platelet count or
endoscopic appearance.
FC levels were elevated more frequently
in IBD patients (89\% vs $9 \%$ ). ASCA
antibodies were detected in $67 \%$ of
patients with CD, The combination of
the measurements of FC and serological
responses to microbial antigens (ASCA,
I2, and OmpW) identified 100\% of CD
patients (specificity $36 \%$ ) and $89 \%$ of UC
patients (specificity $36 \%$ ).

A normalised FC level at the end of the
study predicted a complete response in
$100 \%$ patients, whereas elevated FC level
predicted incomplete response in $30 \%$.
Normalised MPO or EPX levels predicted
a complete response in $100 \%$ and $90 \%$
of the patients, respectively. However,
elevated MPO or EPX levels predicted
incomplete response in $23 \%$ and $22 \%$,
respectively.
In patients with mild to moderately
clinically active disease, FC and FL
identified individuals with and without
recurrent IBD. Faecal markers were more
accurate at predicting clinical disease
activity than CRP, platelet count or
endoscopic appearance.
FC levels were elevated more frequently
in IBD patients (89\% vs $9 \%$ ). ASCA
antibodies were detected in $67 \%$ of
patients with CD, The combination of
the measurements of FC and serological
responses to microbial antigens (ASCA,
I2, and OmpW) identified 100\% of CD
patients (specificity $36 \%$ ) and $89 \%$ of UC
patients (specificity $36 \%$ ).

A normalised FC level at the end of the
study predicted a complete response in
$100 \%$ patients, whereas elevated FC level
predicted incomplete response in $30 \%$.
Normalised MPO or EPX levels predicted
a complete response in $100 \%$ and $90 \%$
of the patients, respectively. However,
elevated MPO or EPX levels predicted
incomplete response in $23 \%$ and $22 \%$,
respectively.
In patients with mild to moderately
clinically active disease, FC and FL
identified individuals with and without
recurrent IBD. Faecal markers were more
accurate at predicting clinical disease
activity than CRP, platelet count or
endoscopic appearance.
FC levels were elevated more frequently
in IBD patients (89\% vs $9 \%$ ). ASCA
antibodies were detected in $67 \%$ of
patients with CD, The combination of
the measurements of FC and serological
responses to microbial antigens (ASCA,
I2, and OmpW) identified 100\% of CD
patients (specificity $36 \%$ ) and $89 \%$ of UC
patients (specificity $36 \%$ ).

A normalised FC level at the end of the
study predicted a complete response in
$100 \%$ patients, whereas elevated FC level
predicted incomplete response in $30 \%$.
Normalised MPO or EPX levels predicted
a complete response in $100 \%$ and $90 \%$
of the patients, respectively. However,
elevated MPO or EPX levels predicted
incomplete response in $23 \%$ and $22 \%$,
respectively.
In patients with mild to moderately
clinically active disease, FC and FL
identified individuals with and without
recurrent IBD. Faecal markers were more
accurate at predicting clinical disease
activity than CRP, platelet count or
endoscopic appearance.
FC levels were elevated more frequently
in IBD patients (89\% vs $9 \%$ ). ASCA
antibodies were detected in $67 \%$ of
patients with CD, The combination of
the measurements of FC and serological
responses to microbial antigens (ASCA,
I2, and OmpW) identified 100\% of CD
patients (specificity $36 \%$ ) and $89 \%$ of UC
patients (specificity $36 \%$ ).

A normalised FC level at the end of the
study predicted a complete response in
$100 \%$ patients, whereas elevated FC level
predicted incomplete response in $30 \%$.
Normalised MPO or EPX levels predicted
a complete response in $100 \%$ and $90 \%$
of the patients, respectively. However,
elevated MPO or EPX levels predicted
incomplete response in $23 \%$ and $22 \%$,
respectively.
In patients with mild to moderately
clinically active disease, FC and FL
identified individuals with and without
recurrent IBD. Faecal markers were more
accurate at predicting clinical disease
activity than CRP, platelet count or
endoscopic appearance.
FC levels were elevated more frequently
in IBD patients (89\% vs $9 \%$ ). ASCA
antibodies were detected in $67 \%$ of
patients with CD, The combination of
the measurements of FC and serological
responses to microbial antigens (ASCA,
I2, and OmpW) identified 100\% of CD
patients (specificity $36 \%$ ) and $89 \%$ of UC
patients (specificity $36 \%$ ).

A normalised FC level at the end of the
study predicted a complete response in
$100 \%$ patients, whereas elevated FC level
predicted incomplete response in $30 \%$.
Normalised MPO or EPX levels predicted
a complete response in $100 \%$ and $90 \%$
of the patients, respectively. However,
elevated MPO or EPX levels predicted
incomplete response in $23 \%$ and $22 \%$,
respectively.
In patients with mild to moderately
clinically active disease, FC and FL
identified individuals with and without
recurrent IBD. Faecal markers were more
accurate at predicting clinical disease
activity than CRP, platelet count or
endoscopic appearance.
FC levels were elevated more frequently
in IBD patients (89\% vs $9 \%$ ). ASCA
antibodies were detected in $67 \%$ of
patients with CD, The combination of
the measurements of FC and serological
responses to microbial antigens (ASCA,
I2, and OmpW) identified 100\% of CD
patients (specificity $36 \%$ ) and $89 \%$ of UC
patients (specificity $36 \%$ ).

A normalised FC level at the end of the
study predicted a complete response in
$100 \%$ patients, whereas elevated FC level
predicted incomplete response in $30 \%$.
Normalised MPO or EPX levels predicted
a complete response in $100 \%$ and $90 \%$
of the patients, respectively. However,
elevated MPO or EPX levels predicted
incomplete response in $23 \%$ and $22 \%$,
respectively.
In patients with mild to moderately
clinically active disease, FC and FL
identified individuals with and without
recurrent IBD. Faecal markers were more
accurate at predicting clinical disease
activity than CRP, platelet count or
endoscopic appearance.
FC levels were elevated more frequently
in IBD patients (89\% vs $9 \%$ ). ASCA
antibodies were detected in $67 \%$ of
patients with CD, The combination of
the measurements of FC and serological
responses to microbial antigens (ASCA,
I2, and OmpW) identified 100\% of CD
patients (specificity $36 \%$ ) and $89 \%$ of UC
patients (specificity $36 \%$ ).

A normalised FC level at the end of the
study predicted a complete response in
$100 \%$ patients, whereas elevated FC level
predicted incomplete response in $30 \%$.
Normalised MPO or EPX levels predicted
a complete response in $100 \%$ and $90 \%$
of the patients, respectively. However,
elevated MPO or EPX levels predicted
incomplete response in $23 \%$ and $22 \%$,
respectively.
In patients with mild to moderately
clinically active disease, FC and FL
identified individuals with and without
recurrent IBD. Faecal markers were more
accurate at predicting clinical disease
activity than CRP, platelet count or
endoscopic appearance.
FC levels were elevated more frequently
in IBD patients (89\% vs $9 \%$ ). ASCA
antibodies were detected in $67 \%$ of
patients with CD, The combination of
the measurements of FC and serological
responses to microbial antigens (ASCA,
I2, and OmpW) identified 100\% of CD
patients (specificity $36 \%$ ) and $89 \%$ of UC
patients (specificity $36 \%$ ).

A normalised FC level at the end of the
study predicted a complete response in
$100 \%$ patients, whereas elevated FC level
predicted incomplete response in $30 \%$.
Normalised MPO or EPX levels predicted
a complete response in $100 \%$ and $90 \%$
of the patients, respectively. However,
elevated MPO or EPX levels predicted
incomplete response in $23 \%$ and $22 \%$,
respectively.
In patients with mild to moderately
clinically active disease, FC and FL
identified individuals with and without
recurrent IBD. Faecal markers were more
accurate at predicting clinical disease
activity than CRP, platelet count or
endoscopic appearance.
FC levels were elevated more frequently
in IBD patients (89\% vs $9 \%$ ). ASCA
antibodies were detected in $67 \%$ of
patients with CD, The combination of
the measurements of FC and serological
responses to microbial antigens (ASCA,
I2, and OmpW) identified 100\% of CD
patients (specificity $36 \%$ ) and $89 \%$ of UC
patients (specificity $36 \%$ ).

A normalised FC level at the end of the
study predicted a complete response in
$100 \%$ patients, whereas elevated FC level
predicted incomplete response in $30 \%$.
Normalised MPO or EPX levels predicted
a complete response in $100 \%$ and $90 \%$
of the patients, respectively. However,
elevated MPO or EPX levels predicted
incomplete response in $23 \%$ and $22 \%$,
respectively.
In patients with mild to moderately
clinically active disease, FC and FL
identified individuals with and without
recurrent IBD. Faecal markers were more
accurate at predicting clinical disease
activity than CRP, platelet count or
endoscopic appearance.
FC levels were elevated more frequently
in IBD patients (89\% vs $9 \%$ ). ASCA
antibodies were detected in $67 \%$ of
patients with CD, The combination of
the measurements of FC and serological
responses to microbial antigens (ASCA,
I2, and OmpW) identified 100\% of CD
patients (specificity $36 \%$ ) and $89 \%$ of UC
patients (specificity $36 \%$ ).

A normalised FC level at the end of the
study predicted a complete response in
$100 \%$ patients, whereas elevated FC level
predicted incomplete response in $30 \%$.
Normalised MPO or EPX levels predicted
a complete response in $100 \%$ and $90 \%$
of the patients, respectively. However,
elevated MPO or EPX levels predicted
incomplete response in $23 \%$ and $22 \%$,
respectively.
In patients with mild to moderately
clinically active disease, FC and FL
identified individuals with and without
recurrent IBD. Faecal markers were more
accurate at predicting clinical disease
activity than CRP, platelet count or
endoscopic appearance.
FC levels were elevated more frequently
in IBD patients (89\% vs $9 \%$ ). ASCA
antibodies were detected in $67 \%$ of
patients with CD, The combination of
the measurements of FC and serological
responses to microbial antigens (ASCA,
I2, and OmpW) identified 100\% of CD
patients (specificity $36 \%$ ) and $89 \%$ of UC
patients (specificity $36 \%$ ).

A normalised FC level at the end of the patients.

\author{
Increased levels of \\ serological responses to \\ microbial antigens (ASCA, \\ $\mathrm{I} 2$, and $\mathrm{OmpW}$ ) and $\mathrm{FC}$ are \\ evident in both $\mathrm{CD}$ and $\mathrm{UC}$ \\ patients. The combination \\ of these markers provides \\ valuable, noninvasive tools \\ for the diagnosis of IBD.
}


Table 1 - Some recent studies about the fecal markers in the evaluation of IBD (cont.)

\begin{tabular}{|c|c|c|c|}
\hline Study & Aim & Patient & Result \\
\hline $\begin{array}{l}\text { Walkiewicz et } \\
\text { al }^{70}\end{array}$ & $\begin{array}{l}\text { To compare FC levels in } \\
\text { IBD and healthy controls, } \\
\text { to correlate FC levels with } \\
\text { clinical disease activity, }\end{array}$ & $\begin{array}{l}32 \text { IBD patients and } 34 \\
\text { healthy controls }\end{array}$ & $\begin{array}{l}\text { The IBD group had higher FC levels } \\
\text { compared with control. Among those } \\
\text { with clinical relapse, } 90 \% \text { had FC levels } \\
\text { more than } 400 \text { mug/g in CD. Eighty-nine } \\
\text { percent of CD encounters with FC levels } \\
\text { less than } 400 \text { mug/g remained in clinical } \\
\text { remission. }\end{array}$ \\
\hline
\end{tabular}

\begin{tabular}{|c|c|c|c|c|}
\hline Xiang et al $^{71}$ & $\begin{array}{l}\text { To investigate possibility and } \\
\text { clinical application of FC in } \\
\text { determining disease activity } \\
\text { of UC }\end{array}$ & $\begin{array}{l}66 \mathrm{UC} \text { and } 20 \text { control } \\
\text { patients }\end{array}$ & $\begin{array}{l}\text { The FC concentration in the patients } \\
\text { with active UC was significantly higher } \\
\text { than inactive UC which was higher than } \\
\text { the control group. There was a strong } \\
\text { correlation between the FC concentration } \\
\text { and the endoscopic gradings for UC. }\end{array}$ & $\begin{array}{l}\text { FC can reflect the disease } \\
\text { activity of UC and can be } \\
\text { used as a rational marker for } \\
\text { intestinal inflammation in } \\
\text { clinical practice. }\end{array}$ \\
\hline Ho et $\mathbf{a l}^{72}$ & $\begin{array}{l}\text { To investigate FC as a } \\
\text { biomarker in predicting } \\
\text { the clinical course of acute } \\
\text { severe UC }\end{array}$ & $\begin{array}{l}90 \text { patients with acute } \\
\text { severe UC requiring } \\
\text { intensive in-patient } \\
\text { medical therapy }\end{array}$ & $\begin{array}{l}\text { FC was significantly higher in patients } \\
\text { requiring colectomy, with a trend } \\
\text { toward significance when comparing } \\
\text { corticosteroid nonresponders and } \\
\text { responders, as well as between infliximab } \\
\text { nonresponders and responders }\end{array}$ & $\begin{array}{l}\text { FC levels are dramatically } \\
\text { elevated in severe UC. } \\
\text { This biomarker can predict } \\
\text { response to first or second- } \\
\text { line medical therapy in this } \\
\text { setting. }\end{array}$ \\
\hline Sipponen et $\mathbf{a l}^{73}$ & $\begin{array}{l}\text { To study the correlation } \\
\text { of FC and FL with simple } \\
\text { endoscopic score for Crohn's } \\
\text { disease (SES-CD) and } \\
\text { histology. }\end{array}$ & $\begin{array}{l}24 \mathrm{CD} \text { patients with } 87 \\
\text { ileocolonoscopies }\end{array}$ & $\begin{array}{l}\text { In ileocolonic or colonic disease, both FC } \\
\text { and FL correlated significantly with colon } \\
\text { SES-CD and colon histology. In patients } \\
\text { with normal FC or FL levels, endoscopic } \\
\text { and histology scores were significantly } \\
\text { lower than in those with elevated } \\
\text { concentrations. }\end{array}$ & $\begin{array}{l}\text { In ileocolonic and colonic } \\
\text { disease, endoscopic score } \\
\text { SES-CD and histological } \\
\text { findings correlated } \\
\text { significantly with FC and } \\
\text { FL. }\end{array}$ \\
\hline Langhorst et $\mathbf{a l}^{74}$ & $\begin{array}{l}\text { To compare the } \\
\text { performance of FL, FC, } \\
\text { polymorphonuclear } \\
\text { neutrophil elastase (PMN-e), } \\
\text { and CRP in patients with } \\
\text { IBD to address whether these } \\
\text { markers can differentiate IBD } \\
\text { patients with endoscopically } \\
\text { assessed inflammation;and } \\
\text { they correlate with } \\
\text { endoscopic severity of } \\
\text { inflammation }\end{array}$ & $\begin{array}{l}54 \mathrm{IBS}, 42 \mathrm{UC}, 43 \mathrm{CD} \\
\text { patients }\end{array}$ & $\begin{array}{l}\text { UC or CD patients with active } \\
\text { inflammation demonstrated significantly } \\
\text { higher levels of FL, FC, and PMN-e } \\
\text { in feces as well as serum-CRP when } \\
\text { compared to patients with inactive } \\
\text { inflammation and patients with IBS. FC } \\
\text { showed the highest diagnostic accuracy } \\
\text { in CD }(81.4 \%) \text {, whereas FL was superior } \\
\text { to the other markers in UC ( } 83.3 \%) \text {. The } \\
\text { comprehensive activity index yielded a } \\
\text { further improvement of sensitivity and } \\
\text { specificity, with a diagnostic accuracy of } \\
95.3 \% \text { for UC patients. }\end{array}$ & $\begin{array}{l}\text { The fecal markers FL, FC, } \\
\text { and PMN-e are able to } \\
\text { differentiate active IBD from } \\
\text { inactive IBD as well as from } \\
\text { IBS. }\end{array}$ \\
\hline
\end{tabular}

FC: Fecal calprotectin, IBD:Inflammatory bowel disease, IBS: Irritable bowel syndrome, ESR: Erythrocyte sedimentation rate, CRP: C-reactive protein, UC: Ulcerative colitis, CD: Crohn disease, CAI: Clinical activity index, FL: Fecal lactoferrin, IC: Indetermined colitis.

Dai et al. studied a total of 177 fresh stool samples collected from 42 active UC, 17 inactive UC, 13 active $\mathrm{CD}, 5$ inactive $\mathrm{CD}, 41$ infectious bowel diseases, 25 IBS and 34 healthy volunteers to evaluate the relationship between fecal lactoferrin and intestinal inflammation by quantitative analysis. Fecal lactoferrin was found to be significantly higher in active IBD than in inactive IBD, IBS and infectious bowel disease. The sensitivity and specificity of fecal lactoferrin for UC were $92 \%$ and $88 \%$, respectively, and for CD were $92 \%$ and $80 \%$, respectively. As a result of this study, fecal lactoferrin was found to be a sensitive and specific marker in measuring the activity of IBD and a valid method for discriminating between inflammatory and noninflammatory bowel diseases. ${ }^{42}$
Kane et al. compared $104 \mathrm{CD}, 80 \mathrm{UC}$ and $31 \mathrm{IBS}$ patients with 56 healthy controls to determine the sensitivity and specificity of fecal lactoferrin concentrations for IBD or IBS. The study found that fecal lactoferrin was $90 \%$ specific for identifying inflammation in patients with active IBD, and elevated levels of lactoferrin were $100 \%$ specific in ruling out IBS. ${ }^{43}$

Schopper et al. studied 64 patients with IBD (36 CD, $28 \mathrm{UC}), 30$ with IBS and 42 healthy controls to determine the accuracy of fecal markers, CRP, blood leukocytes and antibody panels for discriminating IBD from IBS. In addition to CRP and blood leukocytes, blinded fecal samples were measured for calprotectin (PhiCal Tesr, ELISA), lactoferrin (IBD-SCAN, ELISA), Hexagon- 
OBTI (immunochromatographic test for detection of human hemoglobin), and LEUKO-TEST (lactoferrin latex-agglutination test). Also, the blinded serum samples were measured for the ASCA (ELISA) and pANCA (immunofluorescence) antibodies. The authors found that fecal calprotectin and lactoferrin could accurately discriminate between IBD and IBS. Moreover, there was only a marginal improvement in diagnostic accuracy when ASCA and pANCA were also involved. ${ }^{44}$

Another study of 20 patients with IBS, 36 with IBD (24 CD, 12 UC) and 18 with other forms of colitis ( 8 infectious colitis, 5 ischemic colitis, 5 medication-induced colitis) was conducted to evaluate the accuracy of four different fecal markers in discriminating between IBS, IBD and other forms of colitis. In this study, blinded fecal samples were measured for calprotectin ( with PhiCal-Test, ELISA), lactoferrin (with IBD-SCAN, ELISA), with Hexagon OBTI (immunochromatographic test for detection of human hemoglobin) and with LEUKO-TEST (lactoferrin latexagglutination test). The overall accuracies for discriminating IBS from IBD or other forms of colitis were as follows: IBD-SCAN, 91\%; PhiCal-Test, $89 \%$; LEUKO-TEST, 92\%; Hexagon OBTI, 91\%; C-reactive protein, 89\%; and blood leukocytes, $92 \%$. The differentiation of IBD from other forms of colitis usingfecal markers had an overall accuracy ranging from 43 to $50 \%$. The feasibility of fecal sampling in outpatients was high, with an acceptance rate of $95 \%$. In conclusion, the IBD-SCAN and PhiCal-Test had the best overall accuracy for the detection of colitis, followed by the LEUKO-TEST, Hexagon OBTI, C-reactive protein and blood leukocytes. ${ }^{45}$

Fecal lactoferrin might be a helpful noninvasive diagnostic tool for the detection of colitis; however, since it is unspecific, its role in the diagnosis and monitoring of IBD is still questionable. Further studies are necessary to determine its exact place in routine clinical practice.

\section{Fecal Calprotectin}

Calprotectin is a calcium-binding protein that inhibits metalloproteinases, hasantibacterial and antifungal activities and induces apoptosis in malignant and nonmalignant cell cultures. ${ }^{46}$ Calprotectin constitutes $60 \%$ of neutrophil cytosolic proteins and is an abundant protein found in all body fluids in proportion to the degree of inflammation. Calprotectin has many clinical advantages. It is resistant to bacterial degradation in the gut and is stable in stool for up to one week at room temperature, allowing delays in transporting the sample to the laboratory. Furthermore, calprotectin can be readily quantified using ELISA. Notably, random stool samples of $<5 \mathrm{~g}$ show calprotectin concentrations equivalent to 24-hour homogenized specimens, demonstrating that calprotectin is uniformly scattered throughout the feces. ${ }^{47}$

Since calprotectin is primarily derived from neutrophils, its concentration is directly proportional with neutrophil migration toward the intestinal tract. Many studies have dealt with the role of calprotectin in IBD diagnosis and follow-up (Table 1). The leukocyte proteins calprotectin, lactoferrin, lysozyme, myeloperoxidase, and PMN-elastase were compared in fecal samples of three consecutive feces (e.g., three days) in 40 healthy persons, 39 patients with chronic IBD (21 with CD and 18 with UC) and 40 patients with IBS. From this comparison, levels of all of the fecal leukocyte markers in IBS were found to be in the range of healthy patients. Moreover, fecal PMN-elastase and calprotectin still differentiated between chronic IBD and IBS and still correlated with the severity of inflammation. ${ }^{34}$

In our study of 65 IBD patients (14 CD and 51 UC) and 20 outpatients diagnosed with IBS according to Roma II criteria, fecal calprotectin was found to be strongly associated with colorectal inflammation, indicating the presence of organic disease. ${ }^{48}$

Another study was conducted to evaluate the correlation between endoscopic disease activity and fecal calprotectin. The results of the Clinical Activity Index (CAI), CRP and blood leukocytes in $134 \mathrm{UC}$ patients found that endoscopic disease activity correlated closest with the presence of calprotectin. The overall accuracy for the detection of endoscopically active diseases (score $>/=4$ ) was $89 \%$ for calprotectin, $73 \%$ for CAI, $62 \%$ for elevated CRP and $60 \%$ for leukocytosis. In conclusion, fecal calprotectin was the only marker that reliably discriminated an inactive disease from mild, moderate and highly active diseases, highlighting its usefulness for monitoring activity. ${ }^{49}$

In a different study of 31 patients diagnosed with $\mathrm{CD}$, the mean calprotectin concentration in the $\mathrm{CD}$ group was statistically higher than that of the IBS patients. A concentration of $16.01 \mathrm{mg} / \mathrm{l}$ calprotectin had $67.7 \%$ sensitivity and $66.7 \%$ specificity in distinguishing between $\mathrm{CD}$ and IBS. In this respect,the assessment of fecal calprotectin concentration might also be useful for differentiating CD from IBSCD and IBS. ${ }^{50}$

Gisbert et al. followed up 163 patients (89 CD, 74 UC) for 12 months who had been in clinical remission for 6 months to determine the role of fecal calprotectin and lactoferrin in the prediction of IBD relapse. The authors reported that 26 patients (16\%) relapsed during follow-up. Calprotectin concentrations in patients who had suffered a relapse were found to be higher than in patients who had not $(239$ +/- 150 versus 136 +/- $158 \mu \mathrm{gg} / \mathrm{g} ; \mathrm{P}<0.001)$. The relapse risk was higher in patients that had high $(>150$ 
$\mu \mathrm{g} / \mathrm{g})$ calprotectin concentrations $(30 \%$ versus $7.8 \%$; $\mathrm{P}<$ $0.001)$ or positive lactoferrin $(25 \%$ versus $10 \%$; $\mathrm{P}<0.05)$. The sensitivity and specificity of fecal calprotectin $(>150$ $\mu \mathrm{g} / \mathrm{g}$ ) to predict relapse were $69 \%$ and $69 \%$, respectively. The corresponding values for lactoferrin were $62 \%$ and $65 \%$, respectively. As a result, it was concluded that the determination of fecal calprotectin and lactoferrin might be useful in predicting an impending clinical relapse, especially during the following 3 months of remission, in both CD and UC patients. ${ }^{51}$

Similarly, in another study with $97 \mathrm{UC}$ and $65 \mathrm{CD}$ patients in clinical remission, a significant correlation was found between a positive calprotectin test and the probability of relapse in UC patients $(\mathrm{P}=0.000)$. However, in CD patients, only cases of colonic CD had a significant correlation between a positive calprotectin test and the probability of relapse $(\mathrm{P}=0.02) .{ }^{52}$ Although fecal calprotectin levels are considered to change with age, 50 $\mu \mathrm{g} / \mathrm{g}$ of the suggested cut-off level is considered to be useful for all age groups over 4 years old. ${ }^{53}$

However, there are 4 main handicaps of fecal calprotectin to be kept in mind:

- In some studies, low-dose aspirin treatment did not increase fecal calprotectin levels, although the use of nonsteroidal anti-inflammatory drugs (NSAIDs) might cause an increase in calprotectin levels due to NSAID-induced enteropathy in patients without IBD..$^{54,55}$

- Any bleeding in the body over $100 \mathrm{ml}$, including menstrual bleedings, might increase fecal calprotectin levels. ${ }^{56}$

- Some authors suggest that, although fecal calprotectin is considered to be evenly distributed, factors other than disease might contribute to the significant intraindividual biological variations of it $^{57}$.

- Since levels of fecal calprotectin increase in any condition that causes neutrophil migration to the gut, including neoplasms and infections, the sensitivity of fecal calprotectin is not as high as desired. Fecal calprotectin is an easy, inexpensive, sensitive and specific way to evaluate IBD. Despite the fact that levels of fecal calprotectin have an important role in diagnosis, follow-up, prediction of relapses and assessment of response to treatment, it still has some disadvantages and can only be used as a complementary test.

\section{Fecal Pyruvate Kinase}

The dimeric isoform of M2-pyruvate kinase (tumor M2PK), suggested to be a marker of colorectal cancer, has also recently been suggested to be a marker of gastrointestinal inflammation. ${ }^{58}$
Jeffery et al. studied 105 gastroenterology outpatients with a possible diagnosis of organic bowel disease and 94 controls to investigate the role of fecal tumor M2-PK in the differentiation of functional disease from organic bowel disease. The sensitivity, specificity and positive and negative likelihood ratios for diagnosis of organic bowel disease were found to be, respectively, 93\%, 92\%, 11.6 and 0.07 for calprotectin, and, respectively, 67\%, 88\% 5.6 and 0.18 for tumor M2-PK. Calprotectin, in combination with tumor M2-PK, had a sensitivity of $64 \%$, a specificity of $98 \%$ and likelihood ratios of 32 and 0.03 . Tumor M2-PK was useful for the differentiation of organic disease from functional bowel disease but had a lower sensitivity, specificity and predictive value than calprotectin. ${ }^{59}$

The clinical value of fecal pyruvate kinase in IBD patients requires further study.

\section{Rectal Nitric Oxide}

Nitric oxide (NO) is an endogenously produced gas with numerous physiological roles. In response to acute proinflammatory cytokines, leukocytes and epithelial cells express inducible nitric oxide synthase (NOS), which leads to the production and accumulation of significant quantities of NO. ${ }^{60}$

The level of rectal NO correlates with disease activity in IBD patients and it markedly decreases in response to antiinflammatory treatment. This minimally invasive and rapid test is shown to be useful for discriminating between active bowel inflammation and IBS. ${ }^{61}$ Reinders et al. also studied 23 healthy volunteers and 32 patients with IBD to compare calprotectin and rectal NO levels. These authors found that patients with IBD had greatly increased NO and calprotectin levels compared to healthy volunteers $(\mathrm{p}<0.001)$. Moreover, there was a weak correlation between rectal NO levels, disease activity and the number of loose stools in IBD patients (Spearman's rho 0.37 and 0.51 , respectively; $\mathrm{p}$ $<0.05$ ); interestingly, there was no correlation between NO and calprotectin levels. ${ }^{62}$

Ljung et al. studied $22 \mathrm{UC}$ and $24 \mathrm{CD}$ patients to explore rectal nitric oxide (NO) as a biomarker for the treatment response in IBD. Patients with active UC and CD displayed markedly increased rectal NO levels compared to the controls. Rectal NO correlated weakly with disease activity in both UC and CD. Interestingly, the patients' refractory to steroid treatment only slightly increased NO levels compared to those with a therapeutic response. In this respect, the rectal NO level might be a useful biomarker for the treatment response in IBD, since low NO levels are predictive of a poor clinical response to steroid treatment. ${ }^{63}$

However, although rectal NO is a minimally invasive 
test and more expensive than many other fecal tests. More studies are necessary to reveal the exact role of rectal NO levels in IBD patients.

\section{Fecal Myeloperoxidase}

Myeloperoxidase, an enzyme that functions in the oxygen-dependent killing of microorganisms, is released from the primary granules of neutrophils during acute inflammation. The concentration of myeloperoxidase is also proportional to the number of neutrophils within that region. ${ }^{64}$

Silberer et al. compared five different leukocyte proteins, calprotectin, lactoferrin, lysozyme, myeloperoxidase and PMN-elastase and determined their levels by immunoassay in the feces of patients with IBD and IBS and of healthy persons. The areas under the ROC curves of PMN-elastase and calprotectin were not significantly different $(p=0.327)$, whereas PMN-elastase or calprotectin vs. the other proteins were significantly different $(\mathrm{p}<0.001)$. The results suggest that fecal PMN-elastase and calprotectin are important for the differentiation of chronic IBD from IBS. The authors also found that PMN-elastase and calprotectin levels were correlated with the endoscopically classified severity of inflammation but not the myeloperoxidase. ${ }^{34}$

However, Peterson et al. found a relationship between fecal myeloperoxidase levels and the histological indices of disease activity in UC. ${ }^{65}$

Similarly, Wagner et al. showed that normalized MPO levels predicted a complete response to treatment to treatmentin $100 \%$ of the patients, as did normalized fecal calprotectin levels. However, elevated MPO levels predicted an incomplete response in $23 \%$ patients. ${ }^{66}$

In this respect, myeloperoxidase might potentially be used as a surrogate marker for a successful treatment outcome in IBD patients, similar to calprotectin. Further investigations are necessary to identify the clinical role of fecal myeloperoxidase in IBD.

\section{Fecal Eosinophil Protein X}

Eosinophil protein $\mathrm{X}(\mathrm{EPX})$ is released by activated eosinophil granulocytes, which are abundant in the mucosa in active IBD ${ }^{67}$ Fecal EPX levels are mainly studied as an indicator of the treatment outcome in relapses of IBD. Wagner et al. showed that normalized EPX levels have predicted a complete response to treatment in $90 \%$; however, an incomplete response was predicted in $22 \%$ of the patients. In this respect, FC and MPO provide superior discrimination compared to EPX in IBD treatment outcome. ${ }^{66}$ Moreover, fecal EPX levels are also beneficial complements to endoscopical and histopathological evaluations in the daily care of patients with UC. ${ }^{65}$ Still, more studies are necessary to reveal the clinical role of fecal EPX in IBD.

\section{CONCLUSION}

Since inflammatory bowel diseases are chronic, fast, easily available and inexpensive noninvasive tests that are sensitive, specific and simple are necessary for diagnosis and follow-up. A differential diagnosis of organic and inorganic diseases is also important since they might have similar symptoms. Along these lines, fecal lactoferrin and calprotectin tests seem to be one step further from other tests with larger number of studies, higher sensitivity and specificity and wider availability.

\section{Take-home points:}

$>$ None of the current commercially available serological biomarker tests can be used by themselvesin clinics for diagnosis and follow up. Instead, the tests are used as an adjunct to endoscopy in diagnosis and prognosis of the disease.

$>$ The erythrocyte sedimentation rate (ESR), white blood cell count and C-reactive protein (CRP) are good predictors of disease activity in irritable bowel diseases (IBDs). However, since they are non-specific, they are sometimes not helpful for the differential diagnosis and follow-up of IBD.

Indium 111-labeled leukocytes are considered to be the gold standard fecal marker of inflammation, with a $97 \%$ sensitivity for the diagnosis of IBD. However, due to their high cost, the exposure to radiation and the need for prolonged fecal collections of 4 days, they are not recommended for routine use.

$>$ Even though fecal $\alpha 1$-antitrypsin and alpha2-macroglobulin are generally accepted as useful markers of IBD, they are not routinely available or cost-effective.

$>$ Fecal lactoferrin might be a helpful as a noninvasive diagnostic tool for the detection of colitis; however, since it is unspecific, its role in diagnosis and monitoring of IBD remains questionable. Fecal calprotectin is an easy, inexpensive, sensitive and specific method with which to evaluate IBD. Although levels of fecal calprotectin are important in all diagnoses, follow-ups, predictions of relapses and assessment of response to the treatment, it still can only be used as a complementary test.

Tumor M2-PK differentiates organic disease from functional bowel disease but has a lower sensitivity, specificity and predictive value than does fecal calprotectin.

Rectal nitric oxide is a minimally invasive test and is more expensive than many other fecal tests. 
Fecal myeloperoxidase and eosinophil protein $\mathrm{X}$ have potential as a surrogate marker for the determination of successful treatment outcomes in IBD patients, similar to calprotectin.
Further studies are necessary to elucidate the exact role of fecal markers in IBD evaluation.

\section{REFERENCES}

1. Wirtz,S, Neurath MF. Mouse models of inflammatory bowel disease, Adv. Drug Deliv. Rev. 2007;59:1073-83.

2. Hildebrand H, Finkel Y, Grahnquist L, Lindholm J, Ekbom A, Askling J. Changing pattern of paediatric inflammatory bowel disease in northern Stockholm 1990-2001. Gut 2003;52:1432-34.

3. Baumgart DC. The Diagnosis and Treatment of Crohn's Disease and Ulcerative Colitis. Dtsch Arztebl Int. 2009;106:123-33.

4. Guindi M, Riddell RH. Indeterminate colitis. J. Clin. Pathol.2004;57:1233-44.

5. Nikolaus S, Schreiber S. Diagnostics of inflammatory bowel disease. Gastroenterology. 2007;133:1670-89.

6. Langhorst J, Elsenbruch S, Koelzer J, Rueffer A, Michalsen A, Dobos GJ. Noninvasive markers in the assessment of intestinal inflammation in inflammatory bowel diseases: performance of fecal lactoferrin, calprotectin, and PMNelastase, CRP, and clinical indices. Am J Gastroenterol. 2008;103:162-9.

7. Van Limbergen J, Russell RK, Nimmo ER, Ho GT, Arnott ID, Wilson DC, et al. Genetics of the innate immune response in inflammatory bowel disease. Inflamm Bowel Dis. 2007; 13:338-55.

8. Bruining DH, Loftus EV. Current and future diagnostic approaches: from serologies to imaging. Curr Gastroenterol Rep.2007;9:489-96.

9. Li X, Conklin L, Alex P. New serological biomarkers of inflammatory bowel disease, World J. Gastroenterol.2008;14:5115-24.

10. Vermeire S, Vermeulen N, Van AG, Bossuyt X., and Rutgeerts P. (Auto) antibodies in inflammatory bowel diseases. Gastroenterol. Clin. North Am. 2008; 37:429-38.

11. Strober W, Fuss IJ, Blumberg RS. The immunology of mucosal models of inflammation, Annu. Rev. Immunol. 2002;20:495-549.

12. Papp M, Altorjay I, Norman GL, Shums Z, Palatka K, Vitalis Z, et al. Seroreactivity to microbial components in Crohn's disease is associated with ileal involvement, noninflammatory disease behavior and NOD2/ CARD15 genotype, but not with risk for surgery in a Hungarian cohort of IBD patients. Inflamm Bowel. Dis. 2007;13,984-92.

13. Papp M, Altorjay I, Dotan N, Palatka K, Foldi I, Tumpek J, et al. New serological markers for inflammatory bowel disease are associated with earlier age at onset, complicated disease behavior, risk for surgery, and NOD2/CARD15 genotype in a Hungarian IBD cohort, Am J Gastroenterol. 2008;103,665-81.

14. Peyrin-Biroulet L, Standaert-Vitse A, Branche J, Chamaillard M. IBD serological panels: facts and perspectives. Inflamm Bowel Dis. 2007;13:1561-6.

15. Ferrante M, HenckaertsL, Joossens M, Pierik M, Joossens S, Dotan $\mathrm{N}$, et al. New serological markers in inflammatory bowel disease are associated with complicated disease behaviour. Gut. 2007;56:1394-403.
16. Joossens M, Van Steen K, Branche J, Sendid B, Rutgeerts P, Vasseur F,et al. Familial aggregation and antimicrobial response dose-dependently affect the risk for Crohn's disease. Inflamm Bowel Dis. 2009 Jun 5.

17. Mokrowiecka A, Daniel P, Słomka M, Majak P, Malecka-Panas E. Clinical utility of serological markers in inflammatory bowel disease. Hepatogastroenterology. 2009; 56:162-6.

18. Anand V, Russell AS, Tsuyuki R, Fedorak R. Perinuclear antineutrophil cytoplasmic autoantibodies and anti-Saccharomyces cerevisiae antibodies as serological markers are not specific in the identification of Crohn's disease and ulcerative colitis. Can J Gastroenterol. 2008;22:336.

19. Russell RK, Ip B, Aldhous MC, MacDougall M, Drummond HE, Arnott ID, et al. Anti-Saccharomyces cerevisiae antibodies status is associated with oral involvement and disease severity in Crohn disease.J Pediatr Gastroenterol Nutr. 2009;48:161-7.

20. Seow CH, Stempak JM, Xu W, Lan H, Griffiths AM, Greenberg GR, et al. Novel anti-glycan antibodies related to inflammatory bowel disease diagnosis and phenotype. Am J Gastroenterol. 2009;104:1426-34.

21. Chen CS, Sullivan S, Anderson T, Tan AC, Alex PJ, Brant SR, Cuffari $\mathrm{C}$, et al. Identification of novel serological biomarkers for inflammatory bowel disease using Escherichia coli proteome chip. Mol Cell Proteomics. 2009;8:1765-76.

22. Hisabe T, Matsui T, Sakurai T, Murakami Y, Tanabe H, Matake H, et al. Anti-Saccharomyces cerevisiae antibodies in Japanese patients with inflammatory bowel disease: diagnostic accuracy and clinical value. J Gastroenterol. 2003;38:121-6.

23. Lawrance IC, Murray K, Hall A, Sung JJ, Leong R. A prospective comparative study of ASCA and pANCA in Chinese and Caucasian IBD patients. Am J Gastroenterol 2004; 99: 2186-94.

24. Osada T, Ohkusa T, Okayasu I, Yoshida T, Hirai S, Beppu K, et al. Correlations among total colonoscopic findings, clinical symptoms, and laboratory markers in ulcerative colitis. J Gastroenterol Hepatol. 2008;23 Suppl 2:S262-7.

25. Solem CA, Loftus EV Jr, Tremaine WJ, Harmsen WS, Zinsmeister AR, Sandborn WJ. Correlation of C-reactive protein with clinical, endoscopic, histologic, and radiographic activity in inflammatory bowel disease. Inflamm Bowel Dis. 2005;11:707-12.

26. Ha JS, Lee JS, Kim HJ, Moon TG, Chang DK, Lee JH, et al. Comparative usefulness of erythrocyte sedimentation rate and C-reactive protein in assessing the severity of ulcerative colitis.Korean J Gastroenterol. 2006;48:313-20.

27. Szkaradkiewicz A, Marciniak R, Chudzicka-Strugała I, Wasilewska A, Drews M, Majewski P, et al. Proinflammatory cytokines and IL-10 in inflammatory bowel disease and colorectal cancer patients. Arch Immunol Ther Exp (Warsz). 2009;57:291-4. 
28. Poullis A, Foster R, Northfield TC, Mendall MA. Review article: faecal markers in the assessment of activity in inflammatory bowel disease. Aliment Pharmacol Ther 2002;16:675-81.

29. Saverymuttu SH, Peters AM, Lavender JP, Pepys MB, Hodgson HJ, Chadwick SV. Quantitative fecal indium 111-labeled leukocyte excretion in the assessment of disease in Crohn's disease.Gastroenterology. 1983;85:1333-9.

30. Karbach U, Ewe K, Bodenstein H. Alpha 1-antitrypsin, a reliable endogenous marker for intestinal protein loss and its application in patients with Crohn's disease. Gut 1983;24:718-23.

31. Becker K, Berger M, Niederau C, Frieling T. Individual fecal alpha 1- antitrypsin excretion reflects clinical activity in Crohn's disease but not in ulcerative colitis. Hepatogastroenterology. 1999;46:2309-14.

32. Becker K, Niederau C, Frieling T. Fecal excretion of alpha 2 macroglobulin: a novel marker for disease activity in patients with inflammatory bowel disease. Z Gastroenterol 1999;37:597-605.

33. Crama-Bohbouth GEBI, Pena AS. Significance of faecal lysozme excretion and alph-1-antitrypsin clearance in the assessment of activity of inflammatory bowel disease. In: Ge B, editor. Activity Related Abnormalities in Inflammatory Bowel Disease. Woerden-Huybregts Press; 1998. p. 89-103.

34. Silberer H, Küppers B, Mickisch O, Baniewicz W, Drescher M, Traber $\mathrm{L}$, et al. Fecal leukocyte proteins in inflammatory bowel disease and irritable bowel syndrome. Clin Lab 2005;51:117-26.

35. Langhorst J, Elsenbruch S, Mueller T, Rueffer A, Spahn G, Michalsen A, et al. Comparison of 4 neutrophilderive proteins in feces as indicators of disease activity in ulcerative colitis. Inflamm Bowel Dis. 2005;11:108591.

36. Saiki T. Myeloperoxidase concentrations in the stool as a new parameter of inflammatory bowel disease. Kurume Med J. 1998;45:69-73.

37. Dwarakanath AD, Finnie IA, Beesley CM, O'Dowd GM, Nash J, Tsai $\mathrm{HH}$, et al. Differential excretion of leucocyte granule components in inflammatory bowel disease: implications for pathogenesis. Clin Sci. 1997;92:307-13.

38. Adeyemi EO, Hodgson HJ. Faecal elastase reflects disease activity in active ulcerative colitis. Scand J Gastroenterol. 1992;27:139-42.

39. Sugi KSO, Hiarata I, Katsu K. Faecal lactoferrin as a marker of disease activity in inflammatory bowel disease: comparison with other neutrophil derived proteins. Am J Gastroenterol 1996;91:927-34.

40. Guerrant RL, Araujo V, Soares E, Kotloff K, Lima AA, Cooper WH, et al. Measurement of fecal lactoferrin as a marker of fecal leukocytes. J Clin Microbiol. 1992;30:1238-42.

41. Angriman I, Scarpa M, D'Incà R, Basso D, Ruffolo C, Polese L, et al. Enzymes in feces: useful markers of chronic inflammatory bowel disease. Clin Chim Acta 2007;381:63-8.

42. Dai J, Liu WZ, Zhao YP, Hu YB, Ge ZZ. Relationship between fecal lactoferrin and inflammatory bowel disease. Scand J Gastroenterol. 2007:42:1440-4.

43. Kane SV, Sandborn WJ, Rufo PA, Zholudev A, Boone J, Lyerly D, et al. Fecal lactoferrin is a sensitive and specific marker in identifying intestinal inflammation.Am J Gastroenterol. 2003;98:1309-14.
44. Schoepfer AM, Trummler M, Seeholzer P, Seibold-Schmid B, Seibold F. Discriminating IBD from IBS: comparison of the test performance of fecal markers, blood leukocytes, CRP, and IBD antibodies. Inflamm Bowel Dis. 2008;14:32-9.

45. Schoepfer AM, Trummler M, Seeholzer P, Criblez DH, Seibold F. Accuracy of four fecal assays in the diagnosis of colitis. Dis Colon Rectum. 2007;50:1697-706.

46. Steinbakk M, Naess-Andresen CF, Lingaas E, Dale I, Brandtzaeg P, Fagerhol MK. Antimicrobial actions of calcium binding leucocyte L1 protein, calprotectin. Lancet. 1990;336:763-5.

47. Røseth AG, Fagerhol MK, Aadland E, Schjønsby H. Assessment of the neutrophil dominating protein calprotectin in feces. A methodologic study. Scand J Gastroenterol 1992;27:793-8.

48. Erbayrak M, Turkay C, Eraslan E, Cetinkaya H, Kasapoglu B, Bektas $\mathrm{M}$. The role of fecal calprotectin in investigating inflammatory bowel diseases. Clinics. 2009;64:421-5.

49. Schoepfer AM, Beglinger C, Straumann A, Trummler M, Renzulli P, Seibold F. Ulcerative colitis: Correlation of the Rachmilewitz endoscopic activity index with fecal calprotectin, clinical activity, c-reactive protein, and blood leukocytes. Inflamm Bowel Dis. 2009 May 21

50. Eder P, Stawczyk-Eder K, Krela-Ka mierczak I, Linke K. Clinical utility of the assessment of fecal calprotectin in Le niowski-Crohn's disease. Pol Arch Med Wewn. 2008;118:622-6.

51. Gisbert JP, Bermejo F, Pérez-Calle JL, Taxonera C, Vera I, McNicholl $\mathrm{AG}$, et al. Inflamm Bowel Dis. Fecal calprotectin and lactoferrin for the prediction of inflammatory bowel disease relapse.2009;15:1190-8.

52. D'Incà R, Dal Pont E, Di Leo V, Benazzato L, Martinato M, Lamboglia $\mathrm{F}$, et al. Can calprotectin predict relapse risk in inflammatory bowel disease?Am J Gastroenterol. 2008;103:2007-14.

53. Fagerberg UL, Lööf L, Merzoug RD, Hansson LO, Finkel Y. Fecal calprotectin levels in healthy children studied with an improved assay.J Pediatr Gastroenterol Nutr. 2003;37:468-72.

54. Montalto M, Curigliano V, Santoro L, Lombardi M, Covino M, Cammarota G, et al. Prophylactic aspirin therapy does not increase faecal calprotectin concentrations.Eur J Gastroenterol Hepatol. 2006; 18:965-7.

55. Shiotani A, Kamada T, Haruma K. Low-dose aspirin-induced gastrointestinal diseases: past, present, and future.J Gastroenterol. 2008;43:581-8.

56. Røseth AG, Kristinsson J, Fagerhol MK, Schjønsby H, Aadland E, Nygaard K, et al. Faecal calprotectin: a novel test for the diagnosis of colorectal cancer? Scand J Gastroenterol. 1993;28:1073-6.

57. Husebye E, Tøn H, Johne B. Biological variability of fecal calprotectin in patients referred for colonoscopy without colonic inflammation or neoplasm. Am J Gastroenterol. 2001;96:2683-7.

58. Czub E, Herzig KH, Szaflarska-Popawska A, Kiehne K, Socha P, Wo $\mathrm{H}$, et al. Fecal pyruvate kinase: a potential new marker for intestinal inflammation in children with inflammatory bowel disease.Scand $\mathrm{J}$ Gastroenterol. 2007;42:1147-50.

59. Jeffery J, Lewis SJ, Ayling RM. Fecal dimeric M2-pyruvate kinase (tumor M2-PK) in the differential diagnosis of functional and organic bowel disorders. Inflamm Bowel Dis. 2009 May 21. 
60. Lundberg JO, Hellström PM, Fagerhol MK, Weitzberg E, Røseth AG. Technology insight: calprotectin, lactoferrin and nitric oxide as novel markers of inflammatory bowel disease. Gastroenterol Hepatol. 2005;2:96-102.

61. Reinders CI, Herulf M, Ljung T, Hollenberg J, Weitzberg E, Lundberg $\mathrm{JO}$,et al. Rectal mucosal nitric oxide in differentiation of inflammatory bowel disease and irritable bowel syndrome.Clin Gastroenterol Hepatol. 20053:777-83.

62. Reinders CA, Jonkers D, Janson EA, Stockbrügger RW, Stobberingh EE, Hellström PM, et al. Rectal nitric oxide and fecal calprotectin in inflammatory bowel disease. Scand J Gastroenterol. 2007;42:1151-7.

63. Ljung T, Lundberg S, Varsanyi M, Johansson C, Schmidt PT, Herulf M, et al. Rectal nitric oxide as biomarker in the treatment of inflammatory bowel disease: responders versus nonresponders. World J Gastroenterol. 2006;12:3386-92.

64. Peterson CG, Eklund E, Taha Y, Raab Y, Carlson M. A new method for the quantification of neutrophil and eosinophil cationic proteins in feces: establishment of normal levels and clinical application in patients with inflammatory bowel disease. Am J Gastroenterol. 2002;97:1755-62.

65. Peterson CG, Sangfelt P, Wagner M, Hansson T, Lettesjö H, Carlson M. Fecal levels of leukocyte markers reflect disease activity in patients with ulcerative colitis. Scand J Clin Lab Invest. 2007;67:810-20.

66. Wagner M, Peterson CG, Ridefelt P, Sangfelt P, Carlson M. Fecal markers of inflammation used as surrogate markers for treatment outcome in relapsing inflammatory bowel disease. World J Gastroenterol. 2008;14:5584-9.

67. Bischoff SC, Wedemeyer J, Herrmann A, Meier PN, Trautwein C, Cetin Y, et al. Quantitative assessment of intestinal eosinophils and mast cells in inflammatory bowel disease. Histopathology. 1996;28:1-13.
68. Lamb CA, Mohiuddin MK, Gicquel J, Neely D, Bergin FG, Hanson $\mathrm{JM}$, et al. Faecal calprotectin or lactoferrin can identify postoperative recurrence in Crohn's disease.Br J Surg. 2009;96:663-74.

69. Ashorn S, Honkanen T, Kolho KL, Ashorn M, Välineva T, et al. Fecal calprotectin levels and serological responses to microbial antigens among children and adolescents with inflammatory bowel disease. Inflamm Bowel Dis. 2009;15:199-205.

70. Walkiewicz D, Werlin SL, Fish D, Scanlon M, Hanaway P, Kugathasan $\mathrm{S}$. Fecal calprotectin is useful in predicting disease relapse in pediatric inflammatory bowel disease. Inflamm Bowel Dis. 2008;14:669-73.

71. Xiang JY, Ouyang Q, Li GD, Xiao NP. Clinical value of fecal calprotectin in determining disease activity of ulcerative colitis. World J Gastroenterol. 2008;7;14:53-7.

72. Ho GT, Lee HM, Brydon G, Ting T, Hare N, Drummond H, et al. Fecal calprotectin predicts the clinical course of acute severe ulcerative colitis. Am J Gastroenterol. 2009;104:673-8

73. Sipponen T, Kärkkäinen P, Savilahti E, Kolho KL, Nuutinen H, Turunen $\mathrm{U}$, et al. Correlation of faecal calprotectin and lactoferrin with an endoscopic score for Crohn's disease and histological findings. Aliment Pharmacol Ther. 2008;28:1221-9.

74. Langhorst J, Elsenbruch S, Koelzer J, Rueffer A, Michalsen A, Dobos GJ. Noninvasive markers in the assessment of intestinal inflammation in inflammatory bowel diseases: performance of fecal lactoferrin, calprotectin, and PMN-elastase, CRP, and clinical indices. Am J Gastroenterol. 2008;103:162-9. 